\title{
Measurement of Ballooning Gap Size of Irradiated Fuels Using Neutron Radiography Transfer Method and HV Image Filter
}

\author{
Cheul-Muu Sim*', TaeJoo Kim*, Hwa Suk Oh* and Joon Cheol Kim**
}

\begin{abstract}
A transfer method of neutron radiography was developed to measure the size of the end plug and a gap of an intact K102L-2, the irradiated fuel of a ballooned K174L-3, a ballooned and ruptured K98L-3. A typical irradiation time of $25 \mathrm{~min}$. was determined to obtain a film density of between 2 and 3 of SR X-ray film with neutrons of $1.5 \times 10^{11} \mathrm{n} \cdot \mathrm{cm}^{-2}$. To validate and calibrate the results, a RISO fuel standard sample, Cd plate and ASTM-BPI/SI were used. An activated latent image formed in the $100 \mu \mathrm{m}$ Dy foil was subsequently transferred in a dark room for more than 8 hours to the SR film which is a maximum of three half-lives. Due to the L/D ratio an unsharpness of 9.82-14 $\mu \mathrm{m}$ and a magnification of 1.0003 were given. After digitizing an image of SR film, the ballooning gap of the plug was discernible by an $\mathrm{H} / \mathrm{V}$ filter of image processing. The gap size of the ballooned element, K174L-3, is equal to or greater than $1.2 \mathrm{~mm}$. The development of a transfer method played a pivotal role in developing high burn-up of Wolsung and PWR nuclear fuel type.
\end{abstract}

Keywords: Transfer Method, Dy Foil, Neutron Radiography, Irradiated Fuels, Ballooning Gap, HV filter

\section{Introduction}

The non-destructive testing of transfer neutron radiography is performed on nuclear irradiated fuels. The basic difference between neutron radiography and X-ray (or gamma-ray radiography) is their attenuation characteristics inside the object. In the former, it is from a neutron-nucleus interaction, while in the latter the beam attenuation is caused by $\mathrm{X}$-ray-orbital electron interaction in the object, which provides striking difference in their mass attenuation coefficient of the objects. Different isotopes may have a very different neutron cross sections and thus neutron radiography(NR) may reveal the distribution of a particular isotope in a sample[1]. One of the main fields of NR application is the nuclear industry. For example, radiography tests can be conducted for the location of pellets of ${ }^{235} \mathrm{U}$ in fuel elements intended to contain only ${ }^{238} \mathrm{U}$ as well as on the crack, swelling, gap size of plug[2]. Using a transfer exposure method, in which the build-up radioactivity of dysprosium(Dy), indium(In) and gold $(\mathrm{Au})$ screens can be subsequently exposed on a film in a dark room, allowing testing of highly radioactive objects, such as irradiated nuclear fuel. Due to the high radioactivity of a nuclear fuel, a direct method of NR can not be used[1].

The transfer method of neutron radiography is mobilized for an inspection of post irradiated nuclear fuel pins, $\mathrm{U}_{3} \mathrm{Si}$ clad with $\mathrm{Al}$, in which several types of indications of swallowing, crack, ballooning, plug gap and thinning occurred[3-6]. The system of the transfer method consists of a cask for carrying fuel pins, Dy converter, its linear controller and a camera for monitoring a fuel cassette. A type of nuclear fuel pin has been inspected by the transfer method of NR and an autoradiography of SSNTD(solid state nuclear track detector) since

[Received: 2012. 9. 11, Revised: 2013. 2. 4, 2013. 4. 8, Accepted: 2013. 4. 10] *Korea Atomic Energy Research Institute, P.O Box 105 Yuseong, Daejeon, 305-303, Korea, **Seonam University 720 Kwangchi-Dong Namwon Jeonbuk 590-711, Korea †Corresponding Author: cmsim@kaeri.re.kr

(c) 2013, Korean Society for Nondestructive Testing 
the cask for transportation and its manipulation was created in 2005[3-6]. In addition to a precision measurement of the pins structure, the irregularity of the cracks, void, porosity, humidity concentration, corrosion and ballooning are detectable by a transfer method of NR. A transfer method has been developed to inspect the irradiated fuels. To enhance a digital image of a gap in irradiated fuels, which vertically appears, the directional filter method is applied.

\section{Theoretical Background of the Method}

\subsection{Neutron Radiography and Cross Sections}

The detection of defects by radiography is based on the observation of the differences in radiation intensity after passing through the object under examination. This occurs according to the basic law of radiation attenuation. In neutron radiography, a nuclear cross section quantifies the probability that a reaction takes place between the neutron and the target material. This can be considered using a target size measured by $\mathrm{cm}^{2}$. There are several types of cross sections but those of the principal interests to neutron radiographers are the absorptions cross section $\sigma_{a}$ and scattering cross section $\sigma_{s}$. The total cross-section $\sigma_{T}$ is the sum of these two[1].

$$
\begin{aligned}
& \sigma_{a}=\sigma_{r}(n, r)+\sigma \quad(n, p)+\sigma \quad(n, \alpha)+\cdots \\
& \sigma_{s}=\sigma \quad(n, n)+\sigma \quad\left(n, n^{\prime}\right) \cdots \\
& \sigma_{T}=\sigma_{a}+\sigma_{s}
\end{aligned}
$$

where $\gamma=$ gamma,$p=$ proton and $\alpha=$ alphaparticle

The unit of cross section is the barn and its basic unit is $10^{-24} \mathrm{~cm}^{2}$. The neutron transmission through a sample can be expressed by considering the rate at which the neutron intensity reduces as it passes through the sample material. This is given by

$$
\Phi=\Phi_{0} e^{-N \sigma x}
$$

where

$\Phi$ : Neutron transmitted through the sample

$\Phi_{0}$ : Neutrons incident upon the sample

$N$ : Number of target nuclei per unit volume

$\sigma:$ Microscopic cross-section

$x$ : Sample thickness

It should be noted that there are several ways of expressing the cross-section of a material, the microscopic cross-section $\left(\mathrm{cm}^{2}\right)$, macroscopic crosssection $\left(\mathrm{cm}^{-1}\right)$ and mass absorption cross-section $\left(\mathrm{cm}^{2} \mathrm{~g}^{-1}\right)$. The attenuation characteristics are described as following Eq. (5)

$$
\begin{aligned}
& \phi_{1}=\phi_{0}\left(1-\sum_{T} \Delta X_{1}\right) \\
& \phi_{2}=\phi_{0}\left(1-\sum_{T} \Delta X_{2}\right)\left(1-\sum_{T} \Delta X_{1}\right) \\
& \phi_{3}=\phi_{0}\left(1-\sum_{T} \Delta X_{3}\right)\left(1-\sum_{T} \Delta X_{2}\right)\left(1-\sum_{T} \Delta X_{1}\right) \\
& \quad \cdots \cdots \\
& \phi_{n}=\phi_{0}\left(1-\sum_{T} \Delta X_{n}\right)\left(1-\sum_{T} \Delta X_{n-1}\left(1-\sum_{T} \Delta X_{n-2}\right) \cdots\right. \\
& \cdots \cdots\left(1-\sum_{T} \Delta X_{1}\right)
\end{aligned}
$$

Where

$\phi_{0}=$ Neutrons incident upon the sample

$\phi_{1 \sim n}=$ Neutrons transmitted through the sample

$\Delta X_{1}=\Delta X_{2} \cdots \Delta X_{n}$

$\Delta X=$ Thickness

Using binominal theorem, Eq. (5) can be rewritten as.

$$
\Phi=\Phi_{0} \lim _{n \rightarrow \infty}\left(1-\sum_{T} \frac{X}{n}\right)^{n}
$$

Eq. (6) can be rewritten as below

$$
\Phi=\Phi_{0} e^{-\Sigma_{T} X}
$$

The product $N \sigma$ of Eq. (4) is represented by the symbol $\sum_{T}$ in a macroscopic cross-section and is the total area for a given neutron interaction presented by a cubic centimeter of the material. Thus, for this case Eq. (4) can be rewritten as Eq. (7).

The third form, the mass absorption coefficient, is denoted by the symbol $\mu_{m}$ and is related to the macroscopic cross-section by the 
relationship.

$$
\mu_{m}\left(\mathrm{~cm}^{2} \mathrm{~g}^{-1}\right)=\frac{\sum\left(\mathrm{cm}^{-1}\right)}{\rho\left(g \bullet \mathrm{cm}^{-3}\right)}
$$

where $\rho$ is the density $\left(\mathrm{g} \cdot \mathrm{cm}^{3}\right)$. The mass absorption coefficient is the total target area for a given neutron interaction per cubic centimeter of material per unit density. It is therefore more convenient to expresses values for solids and gases, the densities of which normally differ by several orders of magnitude. In this Eq. (4) is expressed as

$$
\Phi=\Phi_{0} e^{-\frac{\sum}{\rho} \cdot x \rho}=e^{-\mu_{m} x \rho}
$$

where $x \rho$ has the dimension of $\mathrm{g} \mathrm{cm}^{-2}$. The values of $\Sigma$ and $\mu_{m}$ are given and an example of the calculation of $\sum$ for a compound is also given. It should be noted that there is a also a cross section called the linear absorption coefficient with the dimension of $\mathrm{cm}^{-1}$.

Assuming that the property of the nuclear species is unaffected by consideration of the molecular or crystal structure in which it resides, the macroscopic cross-section for a compound can be calculated from the summation of the macroscopic cross-section of each nuclear species[1,8].

$$
\Sigma_{c}=\left(\frac{N_{A} \bullet \rho}{A}\right) \bullet \sigma_{t}
$$

where

$\Sigma_{c}=$ Macroscopic cross section of compound

$\rho=$ Density of compound

$N_{A}=$ Avogadro's numbers

$A=$ Atomic weight of compound

$\sigma_{t}=$ Microscopic cross-section of each atom, $\mathrm{cm}^{-2}$

\subsection{Transfer Method}

The transfer method is based on factors influencing the activation of the detection materials by exposure to a neutron beam. The activity produced will be as follows[7]

$$
A=n v \sigma N\left(1-e^{-\lambda t}\right)
$$

$A$ : Activity produced (disintegration/sec)

$n v$ : Neutron flux density

$N$ : Number of inert stable atoms of the isotope to be activated $\left(N={ }^{w g t} /\right.$ atomic_wgt $\left.\times 6.02 \times 10^{23}\right)$

$t=$ Irradiation time

$\lambda=$ Decay constant

$\sigma=$ Activation cross section

The activity produced is directly proportional to the numbers of neutrons striking the sample. The activity produced in a neutron radiographic transfer detection screen is directly related to the attenuation of the inspection object at each point in the image.

\subsection{Horizontal/Vertical Filter}

Many image enhancement techniques are based on a spatial operation performed on the local neighborhoods of the input pixels. Often, the image is convolved with a finite impulse responses filter. Here, each pixel is replaced by a weighted average of its neighborhood pixels, i.e., Eq. (12)

$$
v(m . . n)=\sum_{(K, l)} \sum_{\in w} a(k, l) y(m-k, n-l)
$$

where $y(m, n)$ and $v(m, n)$ are the input and output images, respectively, $w$ is a suitably chosen window, and $a(k, l)$ are filter weights. To protect the edges from blurring while smoothing, a directional averaging filter can be useful. Spatial averages $v\left(m, n: \theta^{H V}\right)$ are calculated in the $\mathrm{H} / \mathrm{V}$ (horizontal/vertical) directions as in Eq. (13).

$$
v\left(m . . n: \theta^{H V}\right)=\frac{1}{N_{\theta}} \sum_{(K, l)} \sum_{\in W} a(k, l) y(m-k, n-l)
$$

A directional $\theta^{H V^{*}}$ of $\mathrm{H} / \mathrm{V}$ is found $\left|y(m, n)-v\left(m, n: \theta^{*}\right)\right|$ that is minimum. The resulting 
of the Eq. (13) is given as Eq. (14)[9].

$$
v(m, n)=v\left(m, n: \vartheta^{*}\right)
$$

\section{Experiment}

3.1 The Equipment Used for the Transfer Method of NR

There are two exposure units along the beam-pass direction. The space of the first exposure unit is $120 \mathrm{~cm}$ in width $\times 185 \mathrm{~cm}$ in length $\times 190 \mathrm{~cm}$ in height. There is a beam channel of $30 \mathrm{~cm} \times 40 \mathrm{~cm}$ between the units through the beam pass line. The exposure time is controlled by the main beam shutter with a $90 \mathrm{~cm}$ thickness located just behind the collimator exit. A quick shutter is installed, which reduces the opening time to 2 seconds. The first exposure unit was located behind the quick shutter, the main beam shutter was designed to test the high-radioactive specimen of the nuclear fuel pins by an indirect, so called, transfer method using a Dy screen converter. There is a hole $30 \mathrm{~cm}$ in diameter and $200 \mathrm{~cm}$ in depth below the floor and a cask is equipped through the ceiling so that a nuclear fuel pin of up to $330 \mathrm{~cm}$ is able to be scanned. The NR test system installed in the exposure room consists of a controller, a linear motion guider, a Dy-converter with an Al. plate, a camera and cask.

\subsection{Standard Sample and Irradiated Nuclear Fuel}

To validate and calibrate the results, a RISO fuel standard sample, Cd and ASTM-BPI (beam purity indicator)/SI(sensitivity indicator) are used $[1,8]$. Visual tests on the three elements of intact K102L-2, irradiated K98L-3 and irradiated K174L-3 taken from KFC-026 were conducted, respectively as shown Table 1 . The design structure of the fuel element is shown in Fig.1.

Table 1 Visual testing on K98L-3, K174L-3 and K102L-2

\begin{tabular}{cccc}
\hline No & Fuel element & Visual Testing & Diameter \\
\hline 1 & K102L-2 & Intact element & $\begin{array}{c}7.87 \pm 0.08 \\
\mathrm{~mm}(\text { Design })\end{array}$ \\
2 & K174 L-3 & $\begin{array}{c}\text { Ballooned } \\
9.13 \mathrm{~mm}\end{array}$ \\
3 & K 98 L-3 & $\begin{array}{c}\text { Ballooned and } \\
\text { ruptured }\end{array}$ & $8.80 \mathrm{~mm}$ \\
\hline
\end{tabular}

\subsection{The Exposure Procedure}

When the transfer method is applied, a typical irradiation time is required to obtain a film darkness density to be between 2 and 3 for Kodak SR film[1,7]. The nuclear sample pins are exposed for 25 minutes at the first neutron exposure room of $10^{7} \mathrm{n} \mathrm{cm}^{2} / \mathrm{sec}$ flux.

1) Attach the Dy screen $(20 \mathrm{~cm} \times 40 \mathrm{~cm})$ on the cassette. Install a cassette on the sample table closely.

2) Position the cask on the ceiling hole on top of the First exposure room

3) Put down the fuel cassette from the cask and adjust the Dy converter through the monitor camera.

4) Exposure.* note : An exposure time of at least $25 \mathrm{~min}$ is appropriate at the first exposure room with neutrons of $1.5 \times 10^{11}$ $\mathrm{n} \cdot \mathrm{cm}^{-2}[1]$.

5) Close the main shutter and the quick shutter.

6) Wait cooling time of more than 5 minutes (very important).

\subsection{The Procedure of Transfer Dy Screen on} Film

1) Lay a foam plastic $(30 \mathrm{~cm} \times 30 \mathrm{~cm})$ on a table.

2) Lay an Al. plate $(30 \mathrm{~cm} \times 40 \mathrm{~cm} \times 0.5 \mathrm{~cm})$ on the foam plastic.

3) Lay 5 sheets of A4 paper on the Al. plate

4) Lay an Al. plate $(20 \mathrm{~cm} \times 40 \mathrm{~cm} \times 0.1 \mathrm{~cm})$ 


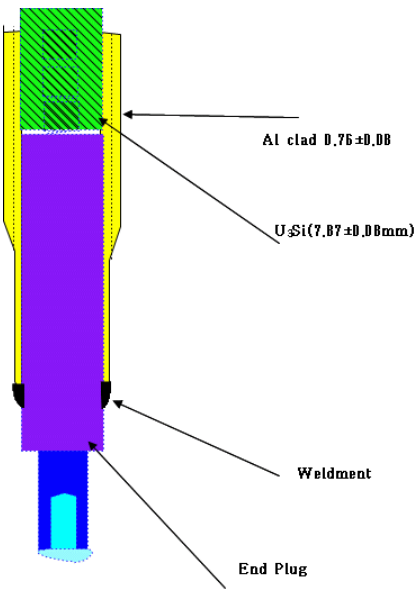

Fig. 1 Fuel element structure

on the A4 paper.

5) Lay 5 sheets of A4 paper on the Al. plate.

6) Lay a Dy screen on A4 paper.

* note: The exposure side shall be up-toward. (very Important)

7) Turn the light off.

8) Lay the SR film on the Dy screen without a lag time

* note: The film coating side shall be down-toward. (very Important)

9) Lay 5 sheets of A4 paper on the film.

10) Lay an Al. plate $(20 \mathrm{~cm} \times 40 \mathrm{~cm} \times 0.1 \mathrm{~cm})$ on the A4 paper.

11) Lay 5 sheets of $\mathrm{A} 4$ paper on the Al. plate.

12) Lay an Al. plate $(30 \mathrm{~cm} \times 40 \mathrm{~cm} \times$ $0.5 \mathrm{~cm})$ on A4 paper.

13) Lay lead bricks neatly on the Al. plate $(30 \mathrm{~cm} \times 40 \mathrm{~cm} \times 0.5 \mathrm{~cm})$.

14) Determine the transfer time.

* note: The Dy transfer time shall be at least 8 hours for maximum of three half-lives[7]

15) Move the film into the developing room.

\section{Result and Discussion}

An unsharpness, $U_{g}$, of the geometrical blur effect is determined by Eq. (15). The diaphragm of inlet beam, $D$, is of $25 \mathrm{~mm}$ in diameter. The length from the aperture to cassette at exposure room 1, $L$, is $5093.8 \mathrm{~mm}$. Thus, $L / D$ of a collimation ratio is converged to 203.7[8]. The distance from image to object, $l$, is assumed to be $2-3 \mathrm{~mm}$. An unsharpness $U_{g}$ is, therefore, approximated to about $9.82 \mu \mathrm{m} \sim 14 \mu \mathrm{m}$. Image magnification, $M$, is 1.0003 by Eq. (16)[8].

$$
\begin{aligned}
& U_{g} \cong \frac{1}{L / D} \\
& M=\left(1-\frac{1}{L}\right)
\end{aligned}
$$

To determine the actual size of the fuel ballooning gap, it is necessary to consider the degree of magnification arising from the collimation of the beam. A Cd-specimen of $10.30 \mathrm{~mm}$ (length) $\times 10.60 \mathrm{~mm}$ (width) is attached to a Dy converter for checking the degree of magnification. A $\mathrm{Cd}$ film image measured with a Nikon projector is approximately magnified into $10.303 \mathrm{~mm}$ (length) $\times$ $10.603 \mathrm{~mm}$ (width) with a round off. A magnification ratio, $M$ of 1.0003 is sufficiently low and can be regarded as negligible. After a digital image of SR film using EPSON 4990 DPI, the gap size of ballooned element of K174L-3 and ballooned/ruptured element K98L-3 was measured using ROI(Region of interest) image, noise removal (using median filter preserving edges) method, enhancement of selected image using histogram equalization method, sobel operator method and Laplacian of Gaussian method in Fig. 3(a-d)[9]. Its size is equal to or greater than $1.2 \mathrm{~mm}$ in Fig. 3(e). It was proved by a post irradiation examination revealed almost the same size shown in Fig. 4. From a view of the image density of ballooned element K174L-3 and ballooned/ruptured element K98L-3, there is no evidence of water in the $1.2 \mathrm{~mm}$ gap, which implies that the ballooning and rupture was not likely owing to water ingress from a leak path through the cladding, end weld or end plug. 


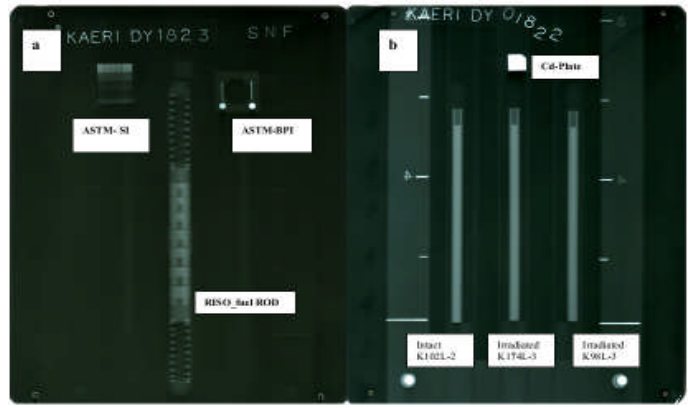

Fig. 2 (a) RISO fuel standard sample, ASTM SI/BPI/ (b) Irradiated fuel of SR film: Intact K102L-2(left), K174L-3(middle), K98L-3(right)
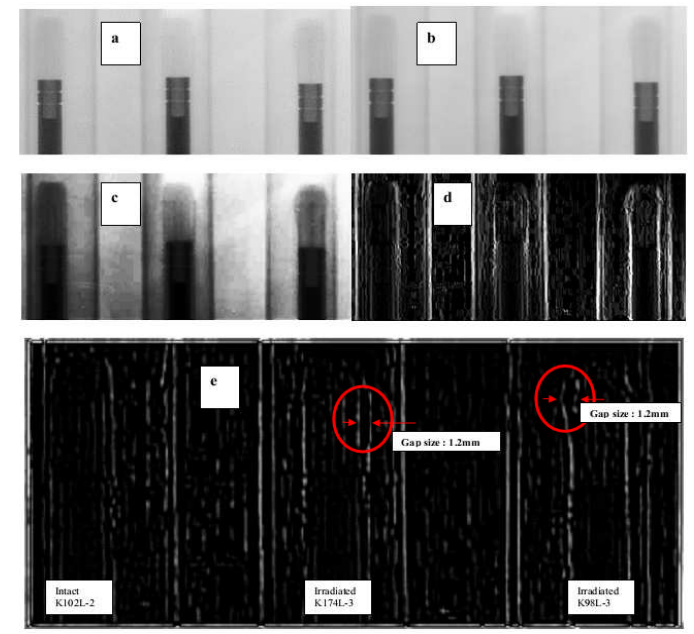

Fig. 3 (a) ROI(region of interest) image, (b) Noise removed image(using Median Filter preserving edges), (c) Enhancement of Selected Image (Histogram Equalization Method), (d) Sobel operator image(using vertical filter-1), (e) Laplacian Gaussian image on K102L-2(left), K174L-3 (middle) and K98L (right)

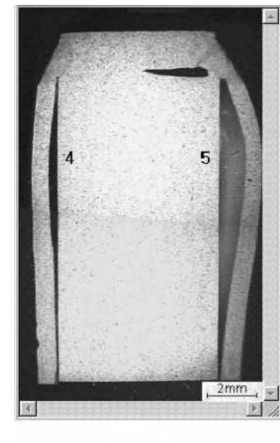

K174L-3(midde)

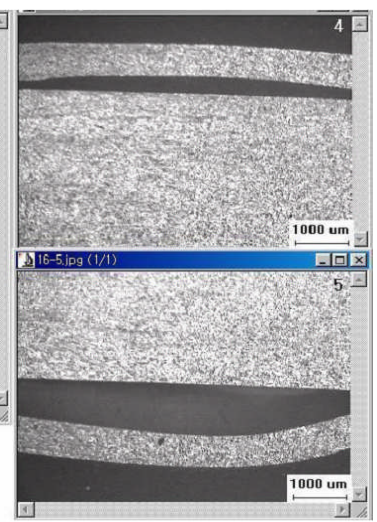

Fig. 4 Metallographic examination results on post irradiated examination conducted on fuel element K174L-3(middle)

\section{Conclusion}

Based on this study, following can be concluded.

1) The technique of the transfer method of NR accompanied with its procedures and system was developed for an inspection of irradiated nuclear fuels.

2) The transfer method of a neutron radiography was carried out to measure the size of the fuel end plug and gap of an intact K102L-2, ballooned K174L-3 and ballooned/ruptured K98L-3, respectively.

3) The gap size of ballooned elements K174L-3 and ballooned/ruptured K98L-3 are equal to or greater than $1.2 \mathrm{~mm}$ based on an image processing of a vertical/horizontal filter. This is almost an identical quantity to the size of the post irradiation examination of K174L-3 and K98L-3.

4) A directional filter of an image processing is a potential tool for measuring the gap of the clad and axial cracks.

5) The development of a transfer method plays a pivotal role in developing the high burn-up of Wolsung and PWR nuclear fuels type.

\section{Acknowledgments}

We thank Mr. Kye Hong Lee and Dr. In Cheol Lim for their support at the HANARO research reactor $(30 \mathrm{MW})$ and IMEF (irradiated material examination facility). The project was funded by KNRF/KAERI nuclear program.

\section{References}

[1] J. C. Domanus, Commission of the European Communities and Euratom Neutron Radiography Working Group, "Practical Neutron Radiography," Commission of European Communities, Klumer Academic Publishers, 
Dordrecht, Boston (1992)

[2] J. C. Domanus, "Reference Neutron Radiography of Nuclear Reactor Fuel," Commission of European Communities, D. Reidel Publishing Company (1981)

[3] F. Groeschel, P. Schleuniger, A. Hermann, E. Lechmann and L. Wiezel, "Neutron radiography of irradiated fuel rod segments at the SINQ: loading, transfer and irradiation concept," Nuclear Instruments and Methods in Physics Research, A 424, pp. 215-220 (1999)

[4] E. Lechmann, P. Vontobel, L. Wiezel, "The investigation of highly activated samples by neutron radiography at the spallation neutron source SINQ," Non-destructive Testing\& Evaluation, Vol. 16, Issue 2-6, pp. 203-214 (2001)

[5] E. H Lehmann, P. Vontobel and A. Hermann, "Non-destructive analysis of nuclear fuel by means of thermal and cold neutrons," Nuclear Instruments and Methods in Physics Research A, 515, pp. 745-759 (2003)

[6] Masayoshi Tamaki, Kazuhiro Lida, Noriaki Mori, Eberhard H. Lechmann, Peter Vontobel and Mirko Estermann, "Dy-IP characterization and its application for experimental neutron radiography tests under realistic conditions," Nuclear Instruments and Methods in Physics Research A, 542, pp. 320-323 (2005)

[7] H. Berger, "Neutron radiography-methods, capabilities and application," Industrial Quality Inc USA, p. 64 (1995)

[8] M. Tamaki, M. Oda, K Takahashi, W. Tanimoto and T. Funahashi, "Study on neutron imaging techniques and processing for developing quantitative neutron radiography," Nuclear Instruments and Methods in Physics Research A, 377, pp. 102-106 (1996)

[9] M. Sonka, "Image Processing, Analysis, and Machine Vision," PWS, ch. 4 (1998) 